\title{
A Health Informatics Reporting System for Technology Illiterate Workforce Using Mobile Phone
} \author{
Alessia Amelio 4 \\ ${ }^{1}$ Department of Computer Science and IT, The University of Lahore, \\ Lahore, Pakistan \\ 2 Department of Computer Science and Engineering, University of \\ Engineering and Technology, Lahore, Pakistan \\ ${ }^{3}$ Department of Computer Science and IT, The Superior University, \\ Lahore, Pakistan \\ ${ }^{4}$ DIMES University of Calabria, Rende (CS), Italy
}

Muhammad Imran Afzal Durrani ${ }^{1} \quad$ Noman Sohaib Qureshi ${ }^{2} \quad$ Nadeem Ahmad $^{3}$ Tabbasum Naz ${ }^{1}$

Appl Clin Inform 2019;10:348-357.

Address for correspondence Muhammad Imran Afzal Durrani, PhD, MSc, MCS, Department of Computer Science and IT, The University of Lahore, Lahore, Pakistan (e-mail: iadurrani007@gmail.com).

\section{Abstract \\ Keywords \\ - human-computer interaction \\ - user-centered design \\ - interfaces and usability \\ - technologically illiterate workforce \\ - verbal autopsy data collection}

Background The reduction and control over neonatal, infant, and maternal mortality is a collective mission of the World Health Organization under United Nations.

Methods This article summarizes the automation of verbal autopsy reporting for neonatal, infant, and maternal mortality with primary focus on user-centered design for technologically illiterate workforce with minimum available resources. The diminution in neonatal, infant, and maternal deaths is not possible until grassroot level quality data are available for mortality. The estimated data are less effective for developing countries like Pakistan because it has heterogeneous demographic pockets with respect to mortality causes. The Neonatal, Infant, and Maternal Death E-surveillance System is a project in which a real-time reporting system is innovated that is useful in detecting the causes of mortality and effective in adopting appropriate countermeasure policies. In a pilot study, the system was implemented initially in nine districts of Punjab, Pakistan. The initial system was refined after getting detailed feedback from district management staff including Lady Health Workers and Lady Health Supervisors. The refined surveillance system was finally implemented in all 36 districts of Punjab, Pakistan.

Results The results exhibited 31\% improvement in infant data collection and $6 \%$ improvement in maternal data collection regarding mortality.

Conclusion This research will be helpful in achieving the milestone of gathering realtime mortality data from grassroot level using user-centered design methodology.

\section{Background and Significance}

Prevention and control over neonatal, infant, and maternal mortality is a global challenge. Lawn et al $^{1}$ pointed out that $99 \%$ of neonatal and child deaths occur in middle- and low-income countries, while $1 \%$ of overall such deaths are estimated in the high-income countries. However, most of the research is being performed in these $1 \%$ demographic regions.

received

December 5, 2018

accepted after revision

March 28, 2019

To the best of our knowledge, a formal real-time reporting system for neonatal, infant, or maternal mortality with death causes could rarely be found in underdeveloped countries. The absence of any real-time data for births and deaths has created a vacuum for the assessment of the actual situation. Uniform macro level countermeasure policies are not effective in developing countries because there exist heterogeneous demographic pockets with respect to mortality causes. The

(c) 2019 Georg Thieme Verlag KG Stuttgart . New York
DOI https://doi.org/ $10.1055 / \mathrm{s}-0039-1688830$. ISSN 1869-0327. 
countermeasures for neonatal, infant, child, and maternal deaths cannot be achieved and sustained with a modest success rate in developing countries with estimated data. The real-time reporting system will enable the acquisition of the actual quality data. It will be helpful to identify the degree of heterogeneity of causes of neonatal, infant, and maternal mortality in Pakistan. The various local demographic pockets will be identified with respect to mortality causes. Such a database once maintained on actual primary data will help to devise appropriate countermeasure policies.

In this article, we explore a simple reporting mechanism with mobile phone from technology illiterate workforce at grassroot level with minimum available resources. The objective of this research is to identify the real mortality causes from heterogeneous demographic pockets using actual data, and not on the basis of estimated data. In the first phase, the simple reporting interface on conventional mobile phone was tested as the communication medium. The technology illiterate workforce was incorporated in every development phase as per the user-centered design (UCD) approach. The evaluation of every prototype phase was tested with end-user to empower technology illiterate workforce. This enabled them to become completely self-contained for reporting from their community demographic areas. Most of these remote areas had no Internet and modern facilities, except the global system for mobile communication (GSM) network.

In the subsequent sections, the challenges faced due to problem space and user community involved in this work are discussed. Then, an iterative design and implementation of a fully functional prototype with a three-stage user study is presented. The evaluation is performed using functional prototype with initially 70 Lady Health Supervisors (LHSs) from the district Rahim Yar Khan (R.Y.K.), Punjab, Pakistan. The conclusion is presented with design recommendations for future instantiations of the concept of verbal autopsy reporting for technology illiterate users.

\section{Objective}

The World Health Organization (WHO) under the United Nations (UN) charter has started a global program referred to as Millennium Development Goals (MDG) for the betterment of mankind around the globe. The MDG 4 and MDG 5 are related to reduction of child mortality and improvement in maternal health, respectively. The goal of MDG 4 was reduction of under-five child mortality (U5MR) by two-thirds ${ }^{2}$ and the MDG 5 milestone was to reduce children mortality by 75\% during 1990 and 2015. ${ }^{3}$ Various developing countries failed to achieve MDG 4 and 5 targets due to multiple factors. Victora et $\mathrm{al}^{4}$ stated that very few low-income countries will be able to achieve the targets described in MDG 4 or 5 . These factors are diverse in nature including heterogeneous demographic pockets, budgetary constraints, and lack of quality data repository in absence of real-time reporting system to ascertain child and maternal mortality causes.

Pakistan is a country where maternal deaths are increasing while the neonatal deaths are stagnant. Pakistan was reported to have 75 deaths per 1,000 pregnancies and 55 neonatal deaths per 1,000 births. ${ }^{5}$ The research conducted by Pasha et $\mathrm{al}^{6}$ is based on multisite prospective study from six different countries, which includes Pakistan, India, Kenya, Zambia, Guatemala, and Argentina. The observation in different sites revealed that the Pakistani site was deficient prominently with respect to maternal health education and after-birth healthcare facilities. The mortality causes were heterogeneous demographically in all survey sites. The seven sites in six different countries may not be regarded as representatives of countrywide statistics; however, are useful indicators in absence of a robust registration system.

Harrison et $\mathrm{al}^{7}$ also conducted research on maternal, neonatal, and fetal health issues in middle- and low-income countries. The study recorded data of more than 260,000 births and observed neonatal and maternal mortality. The focus of this research was on quantitative aspects of fetal, neonatal, and maternal mortality in association with obstructed labor (OL), prolonged labor, and failure to progress. They performed a population-based observatory research of pregnancy at seven different sites in Argentina, Guatemala, India, Kenya, Pakistan, and Zambia. The authors stated that $\mathrm{OL}$ is found to be a common cause for maternal mortality that accounts for $6 \%$ of maternal deaths.

Medhi et $\mathrm{al}^{8}$ have used low-cost, java-enabled mobile phones in healthcare for combating malnutrition in an Indian rural locality. They have used unsupervised field trials for 3 months with the help of 10 rural health workers. The developed system prototype is named CommCare that reported improvements gained in quality of data, completeness, and timeliness.

Liu et $\mathrm{al}^{9}$ have used verbal autopsy to ascertain causes of children death. They have used the vital registration method to collect data related to child mortality. The authors also have predicted child and neonatal mortality separately for the next 15 years. They found that in 2013 neonatal deaths accounted for $44 \%$ out of the total children mortality, which is 6.3 million. It is found that three major causes are liable in children mortality, preterm birth complications, pneumonia, and intrapartum complications. Verbal autopsy is an effective method to ascertain mortality causes of neonatal and maternal deaths. However, with the transformation layers involved in manual registration of verbal autopsy, the effectiveness and accuracy of the death causes may not be determined.

Hogan et $\mathrm{al}^{10}$ have conducted a systematic analysis for progress on MDG 5 in 181 countries using vital registration, census, surveys, and verbal autopsy. The study findings were that only 23 countries are on track for MDG 5 to achieve a 75\% reduction in maternal mortality and rest of the countries did not achieve the target. Pakistan was among those countries which were unable to decrease maternal mortality, confirmed by the Planning Commission of Pakistan ${ }^{2}$ report.

Verbal autopsy is a common reporting mechanism for neonatal, infant, and maternal mortality. In order to manage this system by technology illiterate workforce responsible for reporting is transparent and has fewer chances of errors. However, the case in developing countries is not identical. The developing countries in general and South Asian countries in particular have a health service infrastructure with 
technologically illiterate workforce at the grassroot level. For semiliterate and technologically illiterate people, the interface for a reporting system cannot be the same as for the literate workforce. $^{11,12}$ The UCD is used for technologically illiterate workforce for reporting neonatal, infant, and maternal mortality. The focus of modern information era is on developing competitive software and less effort and resources are diverted toward UCD for semiliterate or technology illiterate users. This negligence may result in loss of productivity in organizations and increased error rate in day-to-day working. ${ }^{13}$

\section{Methods}

This research was conducted in collaboration with Integrated Reproductive Maternal Newborn Child Health and Nutrition Program (IRMNCH \& NP), Primary \& Secondary Healthcare Department of Government of Punjab, Pakistan, and United Nations Fund for Population Activities (UNFPA), Pakistan. The main objective of IRMNCH \& NP is to decrease newborn, child, and maternal morbidity and mortality, promote family planning services, and expand nutritional supplements status of children and women. The major focus of UNFPA is to support and strengthen family planning services, data gathering, and analysis related to population problems.

To the best of our knowledge, there is no online reporting system for neonatal, infant, and maternal mortality at provincial level where the government policy makers take decisions on real data. Therefore, the Punjab health department needs a centralized death reporting system where it can monitor real-time data collected from health facilities. The health department's primary emphasis is on finding death causes with service delivery gaps of neonatal, infant, and maternal mortality at the health facility and community level. The data collection procedure was slow and time consuming as per previous practice in the manual reporting system. Thus, the maintenance processes were cumbersome to execute with more chances of error. And it is the reason for delayed decisions and not taking corrective measures due to time-consuming manual processes. The online verbal autopsy, Neonatal Infant and Maternal Deaths E-surveillance System (NIMDS), will facilitate higher throughput as compared with manual system by all means. The online centralized reporting will overcome the delays in reporting and will facilitate real-time appropriate policies.

\section{Target Users}

For the initial study, our research subjects were LHSs from health facilities of district R.Y.K. R.Y.K. has diversity in physical geography as it contains a desert area which is called Cholistan and a riverside along with a canal irrigated area. The overall literacy rate of R.Y.K. is $42 \%$ (55\% in males and $29 \%$ in females). With this literacy rate, it comes on 33rd position out of 36 districts of Punjab. ${ }^{14}$ Initially 70 LHSs were involved. Five out of 70 LHSs were from the urban area, that is, $7.1 \%$, while the other 65 are from rural area representing $92.9 \%$ of LHSs. The LHSs had a minimum schooling of 12 years and 16 years as maximum schooling. Furthermore, the age of LHS is between 27 and 49 years giving a range of 22 , with a mean of 38.06 and median of 38.00 . To understand the technological skills of LHSs, a preexperiment questionnaire was given to them. From the analysis of preexperiment questionnaire, it was found that 23 (32.9\%) LHSs regularly use computers at home out of 70 and 30 (42.9\%) LHSs had used a desktop computer once in their life, while 17 (24.3\%) of them never used computer in their lives. All LHSs had personal mobile phones, out of which 55 (78.6\%) LHSs had conventional mobile phones with an average price of $\$ 20$. Fifteen LHSs had personal smartphones representing the rest of $21.4 \%$. Twenty-five (35.7\%) LHSs had used a smartphone at least once in their life. Forty-five (64.3\%) LHSs had no knowledge about smartphones and its applications. Regarding the use of Internet, the study shows that 12 (17.1\%) out of 70 LHSs used mobile Internet. Furthermore, 58 (82.9\%) out of 70 had never used mobile Internet. At the end, it was interesting to describe the purpose of Internet use. Sixty-five (92.9\%) out of 70 did not give any details or never used the Internet. Only one $(1.4 \%)$ used it for games and the rest $4(5.7 \%)$ out of 70 used the Internet for general purposes.

\section{User-Centered Interface Design}

In designing the online verbal autopsy system, the UCD technique is used. The UCD can be characterized by various points of view and places the user at the center. All definitions are described with attention on the user and focusing the user viewpoint in all phases of the design process. Donald Norman defines UCD as "a philosophy based on the needs and interests of the user, with an emphasis on making products usable and understandable." ${ }^{15}$ Each phase of the UCD concentrate on testing and analysis. These activities need to be iterative in terms of the processes they execute. Abras et al $^{16}$ propose that the design should be based on user's perspective rather than the developer's perception of user-centric approach. The main theme should be focused on how the user is more comfortable with the design.

Currently, the real-time reporting system for neonatal, infant, and maternal death is envisioned. This system may be deployed through mobile phone as the communication medium. Considering ease of use from LHSs' perspective, the user design was based on the available resources. In User Interface (UI) designing phase, with the guidelines of UCD and ethnographic techniques: we started discussions and interviews to ascertain the use of technology and understanding the level of LHSs. An 8-hour long introductory session was conducted on how to send text messages, playing games, and using different apps on a smartphone to gather the attention of LHSs. After the session, our results were not up to the mark for developing a smartphone app of verbal autopsy reporting.

Therefore, after the LHSs feedback and consultation with the district management staff including the District Coordinator Officer, Social Organizer, and Assistant District Coordinator Officer, we decided to design a simpler mechanism for verbal autopsy reporting. Again, an informal group session was conducted with LHSs instead of a formal session individually and it was found to be more useful. First, a prototype was designed after an informal group session with LHSs on a conventional mobile phone. The prototype was a short 
message service (SMS) string with seven variables. Each neonatal, infant, and maternal death report has to be written as the text message. If the death of a baby occurs under 28 days of age, the keyword NEONATAL is used, and if the death of a baby occurs over 28 days to under 1 year of age, then the keyword INFANT is used. Similarly, if the death of a women occurs while pregnant or within 42 days of termination of pregnancy, the maternal death report has to be written in the text message area with the keyword MATERNAL. The format of text message should be started with keyword and then with variables where each variable is separated with an asterisk symbol $\left({ }^{*}\right)$. The length of a variable will count from one asterisk symbol $\left({ }^{*}\right)$ to another asterisk symbol including spaces and keyword which is case-sensitive with correct spelling. The proper order of variables is mandatory for successful acceptance at server level. A SMS short-code number is used at the server side to send and receive messages. The capacity of SMS server is 30 to 35 messages per second to send and receive SMS regarding neonatal, infant, and maternal deaths reporting.

For neonatal death, the string format is as follows:

NEONATAL * Name-of-Neonate * Father's-Name * Date-ofDeath * Village/City * District-Name * LHS-Name-with-code For infant death, the string format is as follows:

INFANT * Name-of-Infant * Father's-Name * Date-of-Death

${ }^{*}$ Village/City ${ }^{*}$ District-Name * LHS-Name-with-code

For maternal death, the string format is as follows:

MATERNAL * Name-of-Maternal * Husband's-Name * Dateof-Death* Village/City * District-Name * LHS-Name-withcode

\section{Proposed Process}

Lady Health Workers (LHWs) and community midwives (CMWs) of that area where neonatal, infant, or maternal death occurred inform the respective LHS. After the confirmation of death, an LHS sends a SMS with complete neonatal, infant, or maternal death string to the system from her registered mobile number. If the SMS reporting format is correct, then the system sends an auto confirmation message with a unique Case Number of that death. LHS should maintain a register for each reported death with Case Number and LHWs'“Khandan/ Family Number." On the other side, if the format is incorrect, the system generates an error message with an auto reply to the sender about the specific error string. Also, the duplication entries are handled in two layers, first through an intelligent system: if the name of the decedent, father/husband name, and date of death are matched with respective LHS register mobile number then the system replies a duplication message error: "You have already reported this death" with return of the Case Number that was previously sent by system through auto reply. Second, every death has to be investigated by the LHS in her community area at the decedent home. The LHS is responsible for the administrative and supportive supervision of the community area for primary and secondary healthcare, so the chances of duplicate reporting are minimum. If any duplicate death report is found, then she reports it to the district office and removes it from the online system. In - Fig. 1, the flow of NIMDS is described. The process is repeated until the correct data have been given by the LHS and then the reported data are saved in the database. After that, the LHS visits the locality to fill the basic information on the verbal autopsy form and finally submits it to the district health office.

The main six steps of NIMDS are as follows:

1. LHWs/CMWs will inform the LHS about death in their area/region and the LHS will send a SMS to initiate a case.

2. The LHS will visit the decedent home and perform a physical verbal autopsy on a specific form.

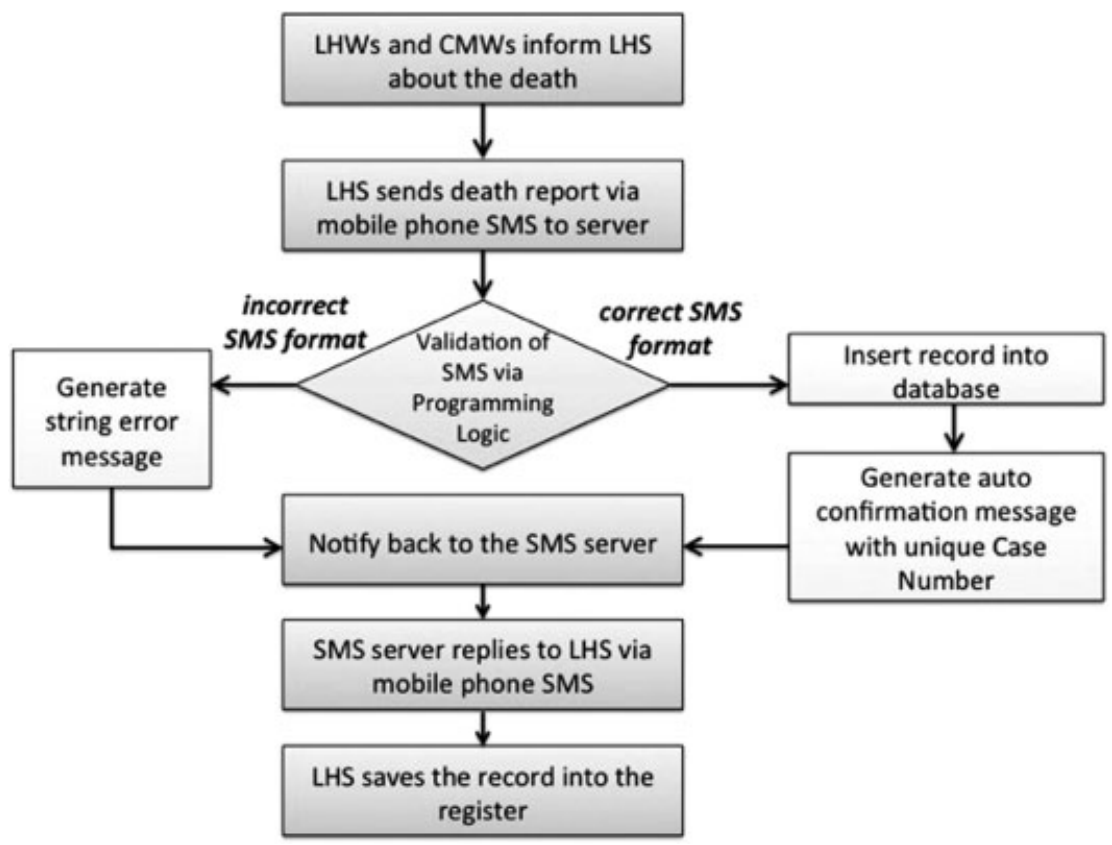

Fig. 1 Death reporting mechanism of the Neonatal Infant and Maternal Deaths E-surveillance System. CMWs, community midwives; LHWs, lady health workers; SMS, short message service. 


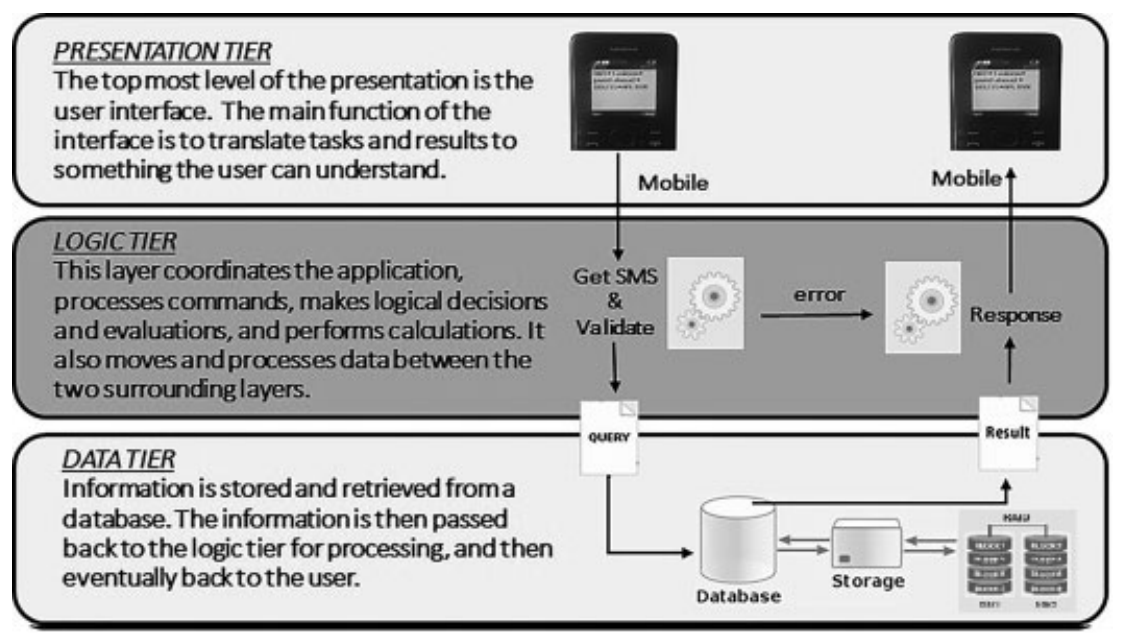

Fig. 2 Neonatal Infant and Maternal Deaths E-surveillance System application: three-tier architecture with redundant array of inexpensive disks storage. SMS, short message service.

3. For the previous month review, every LHS will submit verbal autopsy reports/forms to the district office in the monthly review meeting.

4. District Management Unit (DMU) will submit maternal autopsy forms to the gynecologist and neonatal/infant autopsy forms to the pediatrician for determining obvious and underlying causes of death.

5. DMU computer operator will upload the autopsy forms on the online management information system (MIS) for analysis at the provincial level.

6. The executive district officer (health) will call the district review board meeting to review the verbal autopsy forms with the guidance of the gynecologist/pediatrician.

\section{Web Application Server}

NIMDS's conventional mobile application uses a three-tier architecture (see - Fig. 2): (1) Presentation tier, (2) Logic tier, and (3) Data tier. Presentation tier is the top most level of the application. It is a layer by which LHSs can communicate directly with the system. This tier shows the information that the LHSs use for writing the message to report in their mobile message area with the selected parameters of the decedent. Logical tier is a drag-out from the Presentation tier. It controls the application's functionality by performing detailed processing. Logical tier is the core part of the NIMDS's application, and it has two main components: (1) SMS API, and (2) Validator (programming logic). SMS shortcode API is used for sending and receiving SMS for reporting. The SMS API response time is very high (it can send and receive 35-40 SMSs per second), that is why the system replies to the user in real time. Validator is the programming logic layer which can check the correct SMS format and check that every SMS has come from a valid LHS mobile number or not. Data tier is an element that contains most of the database and instance objects utilized by an application. It accesses the information from the business logic layer. This tier includes the database servers, where data are stored and retrieved in a systematic way.

\section{Pilot Study}

For real-time reporting, the NIMDS was implemented in nine pilot districts of Punjab (i.e., Gujranwala, Jhelum, Narowal, Nankhana Sahib, Okara, Pakpattan, Sahiwal, Sargodha, and Vehari), Pakistan. The initial response was satisfying and the system started to receive continuous streams of data. Within a month of pilot study, the system encountered some problems. LHSs faced difficulty in filling all seven variables, especially in right spellings, format, and true order. The major problems found in the pilot study are as follows:

Problem 1: The SMS keywords were case-sensitive, mostly LHSs type first letter of the keyword in capital letter and others in small letters (i.e., Neonatal, Infant, or Maternal).

Problem 2: Doubt between Newnatal and Neonatal keyword.

Problem 3: Death reports were sent from different mobile numbers which were not saved in the system database.

Problem 4: Not following the proper order of variables.

\section{Revised Prototype with Recommendations}

The following design interface recommendations were accommodated by LHSs feedback:

- Keywords should not be case sensitive.

- Keyword spellings should be abbreviated and easy.

- Type minimum variables information in string.

- They can report from more than one registered mobile numbers in the system.

To resolve these problems in the second prototype, we removed the variables "District-Name" and "LHS-Codewith-Name" because they could be easily identified by our intelligent system. In the new system, the phone numbers of each LHS were linked with their profile and then identified with their respective districts. The keywords were shortened from NEONATAL to NEO, INFANT to INF, and MATERNAL to MAT without case sensitivity which were easy for LHSs to operate. The graphical look of revised death 


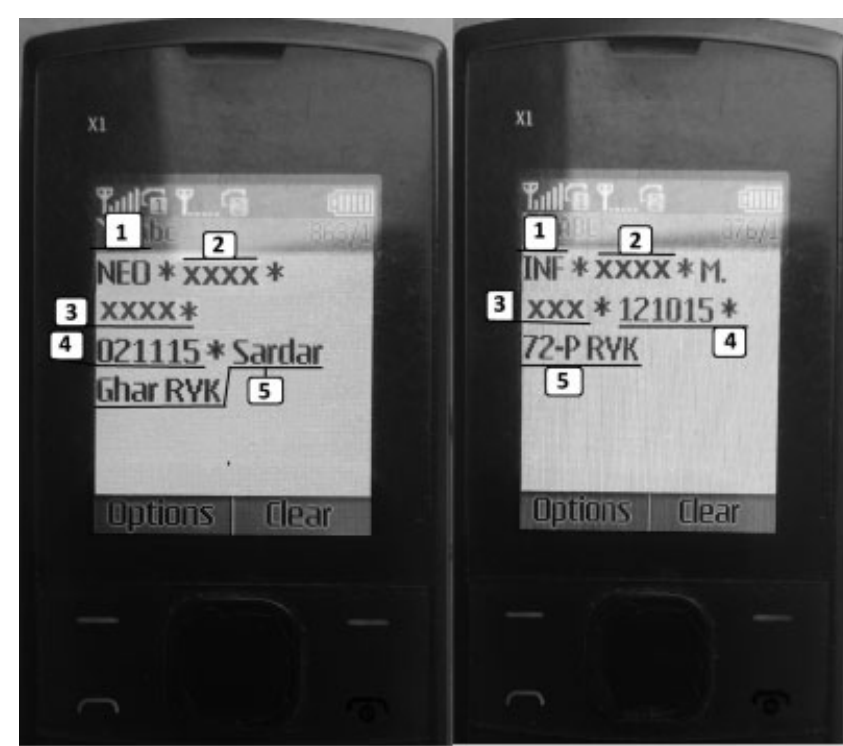

1. SMS Type

4. Date of Death $(021115=2$ nd Nov $2015,121015=12$ th 0

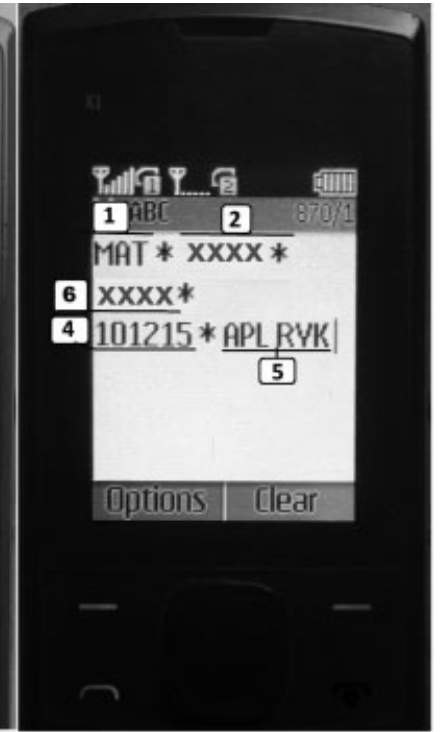

3. Father Name

5. Village / City Name

Fig. 3 Revised neonatal, infant, and maternal reporting in the text message area.

reporting SMS formats are shown in - Fig. 3. Now the LHSs did not have to write a lot of information which resulted in quick and accurate data responses. Also, they can report from maximum of three registered mobile numbers.

The revised format of neonatal death report string is as follows:

NEO * Name-of-Neonate * Father's-Name * Date-of-Death * Village/City

The revised format of infant death report string is as follows:

INF $^{*}$ Name-of-Infant ${ }^{*}$ Father's-Name * Date-of-Death * Village/City

The revised format of maternal death report string is as follows:

MAT ${ }^{*}$ Name-of-Maternal * Husband's-Name * Date-ofDeath * Village/City

\section{User Study}

We conducted the user study in three stages.

\section{Stage 1}

With the help of ethnographic technique it was expected that our target users will be comfortable with smartphone, but most of the LHSs never used a smartphone and were less comfortable with its usage. The reasons are already stated in User-Centered Interface Design section that every ethnographic design is not fit for every problem domain.

\section{Stage 2}

The initial conventional mobile phone reporting interface was formed after getting feedback from technology-illiterate LHSs. The designed system was implemented in district R.Y. K., where 70 LHSs used the system for reporting. However, when the system was implemented for pilot study in 9 districts of Punjab, Pakistan, some problems were found which are already stated in the Pilot Study section.

\section{Stage 3}

UCD iterative process was used for interface refinement. The key lessons that were learned by the UCD iterative process helped in formulating the second prototype of the NIMDS. During ethnographic study and communication with LHSs, it was observed that minimum text string is strongly preferred over longer text and it is less error prone.

\section{Graphical User Interface}

-Fig. 4 shows the graphical UI of the MIS, where the DMU monitors the reported data through SMS in tabular format in real-time.

-Fig. 5 depicts the verbal autopsy data filled by LHS at the time of visiting a decedent home and data with obvious and underlying cause of death written by the gynecologist/ pediatrician after the complete analysis of the verbal autopsy form.

\section{Results}

The primary emphasis of this study was a UCD-specific reporting system that is at the comfort level of all stakeholders, especially for the technologically-illiterate LHSs. The secondary target included the involvement of end users throughout the development process to increase the possibility of enhancing its success. Therefore, we used UCD principles and utilized an assortment of usability-testing techniques to assess the viability of screen design on a conventional mobile phone. The procedures were done in an iterative manner as described in -Fig. 6, until the task objectives have been achieved. 


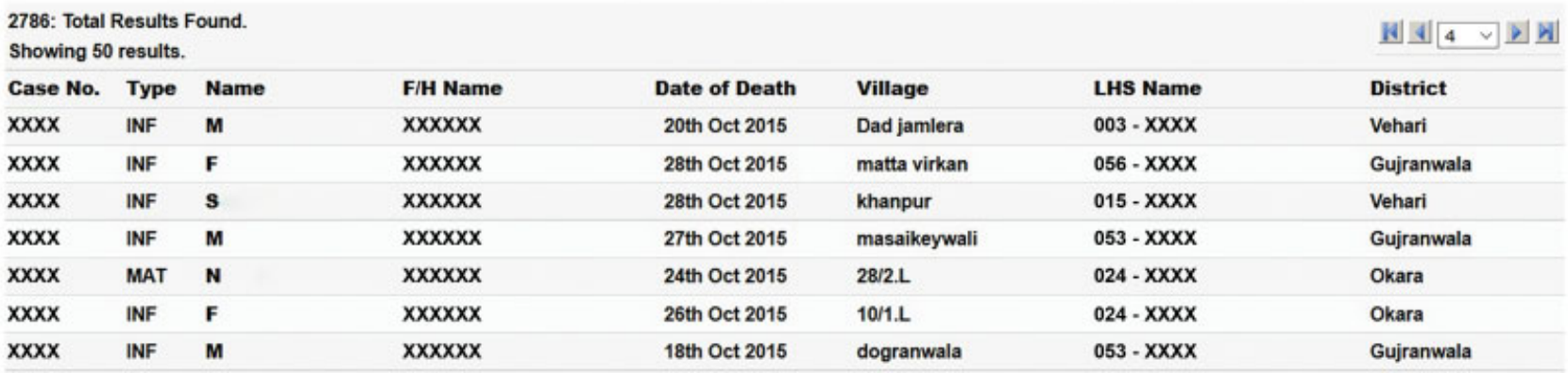

Fig. 4 Graphical user interface-Death short message service data in the management information system.

\begin{tabular}{|c|c|c|c|c|c|c|c|c|c|c|}
\hline \multicolumn{10}{|c|}{$\begin{array}{l}\text { 192: Total Results Found. } \\
\text { Showing } 50 \text { results. }\end{array}$} & \multirow{2}{*}{ 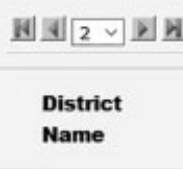 } \\
\hline Case\# & Section & $\begin{array}{l}\text { Place of } \\
\text { Delivery }\end{array}$ & $\begin{array}{l}\text { Delivery } \\
\text { Conducted }\end{array}$ & $\begin{array}{l}\text { Place of } \\
\text { Death }\end{array}$ & $\begin{array}{c}\text { Nature of } \\
\text { Delivery }\end{array}$ & $\begin{array}{c}\text { First } \\
\text { Pregnancy }\end{array}$ & $\begin{array}{c}\text { Age } \\
\text { (in years) }\end{array}$ & $\begin{array}{l}\text { Cause of Death } \\
\text { (Obs=Obvious, } \\
\text { Und=Underlying) }\end{array}$ & Village & \\
\hline$\underline{x x x x}$ & Yes & $\begin{array}{c}\text { Private } \\
\text { Hospital/Clinic }\end{array}$ & Doctor & $\begin{array}{l}\text { Private } \\
\text { Hospital }\end{array}$ & C-section & No & 25 & $\begin{array}{l}\text { Obs:PPH } \\
\text { Und: }\end{array}$ & $x x x x x$ & Sargodha \\
\hline$\underline{\underline{X X X X}}$ & Yes & DHQ & Doctor & DHQ & C-section & No & 32 & $\begin{array}{l}\text { Obs:Pulmonary } \\
\text { Embolism } \\
\text { Und: }\end{array}$ & $x x x x x$ & Vehari \\
\hline$\underline{x x x x}$ & No & THQ & Doctor & $\begin{array}{l}\text { Other Govt } \\
\text { Hospital }\end{array}$ & Episiotomy & No & 27 & $\begin{array}{l}\text { Obs:PPH } \\
\text { Und:APH }\end{array}$ & $x x x x x$ & Rawalpindi \\
\hline$\underline{x x x x}$ & No & Home & Dai & $\begin{array}{l}\text { Other Govt } \\
\text { Hospital }\end{array}$ & Normal & No & 25 & $\begin{array}{l}\text { ObS:POST PARTUM } \\
\text { HAEMORRAGE } \\
\text { Und: }\end{array}$ & $x x x x x$ & Mianwali \\
\hline$\underline{x \times X X}$ & Yes & Govt Hospital & Doctor & $\begin{array}{l}\text { Other Govt } \\
\text { Hospital }\end{array}$ & C-section & No & 33 & $\begin{array}{l}\text { Obs:Cardiac Problem } \\
\text { Und:Cardiac Arrest }\end{array}$ & $x x x x x$ & Rawalpindi \\
\hline$\underline{x X X X}$ & No & $\begin{array}{c}\text { Private } \\
\text { Hospital/Clinic }\end{array}$ & Doctor & $\begin{array}{l}\text { Private } \\
\text { Hospital }\end{array}$ & Normal & No & 42 & $\begin{array}{l}\text { Obs:PI 16, Multigravida } \\
\text { Und:Risk factor }\end{array}$ & $x x x x x$ & Rawalpindi \\
\hline
\end{tabular}

Fig. 5 Graphical user interface-Verbal autopsy data in the management information system with obvious/underlying cause of death written by the gynecologist/pediatrician.

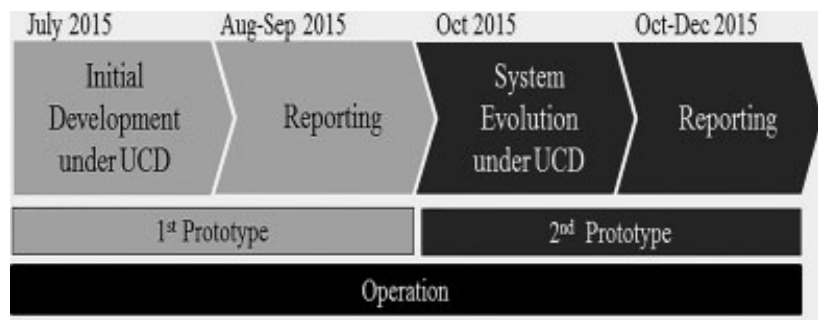

Fig. 6 System development and evaluation process.

Generally, more than 450 LHSs were directly reporting to NIMDS in the pilot study, each LHS required 5 minutes on average in typing the message string of death report in the first prototype, while during the revised final prototype it was observed that the same LHSs were significantly more self-confident and took approximately 1.5 minutes on average to complete the message string of death report. According to LHSs, the reporting mechanism on conventional mobile is very easy and user friendly.

With online verbal autopsy NIMDS, we also continued the collection procedure of manual reporting from districts on monthly basis. This study found a difference of significant number of reported deaths when compared with the NIMDS district-wise reported cases with manual reporting. After the analysis of the pilot study, it is shown that depending on the district NIMDS compared with manual reporting of maternal death is lower from $2.78 \%$ to higher of $25.00 \%$. Mainly, the NIMDS reports have better results establishing an improvement of $6.28 \%$ in the registration of maternal deaths. Furthermore, concerning infant deaths, the NIMDS's improvement is much better. Basically, depending on the district, NIMDS compared with manual reporting of infant death is lower from $7.98 \%$ to higher of $87.04 \%$. In this way, it was absorbed that in NIMDS $31.14 \%$ infant death reported cases were improved. The percentage clearly shows the improvement and real reporting from the community level. The details of reported cases in all the pilot studies in the nine districts are given in - Figs. 7-9.

\section{High Data Efficiency}

As we mentioned previously, the mode of data collection was manual. There were several flaws in data collection, even files were often misplaced or took couple of weeks in reaching the head office. NIMDS has eliminated all dependencies on manual processes and human errors by introducing data flow in real time. Now the data are more secure, efficient, and in unique order where we can find different patterns to identify the causes of death. 


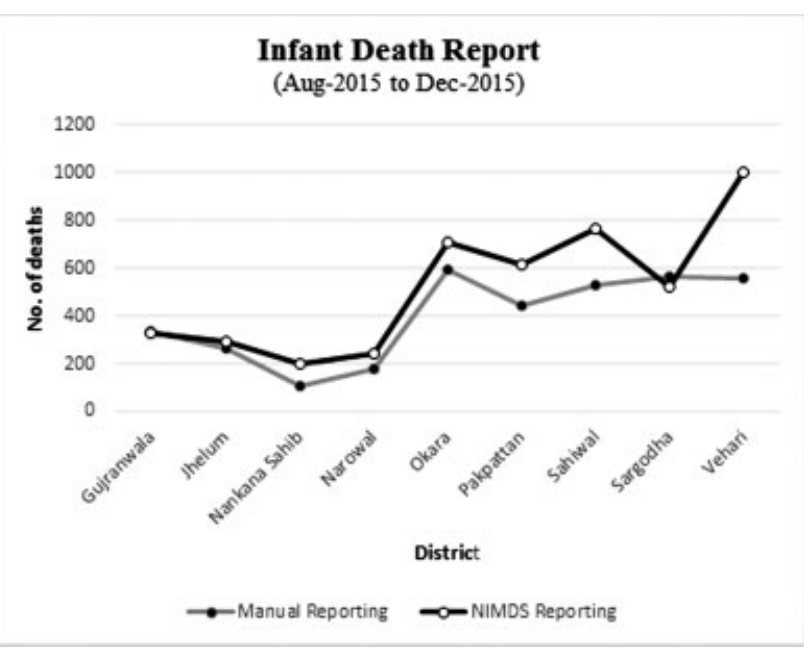

Fig. 7 District-wise infant death comparison between manual reporting and Neonatal Infant and Maternal Deaths E-surveillance System (NIMDS) reporting.

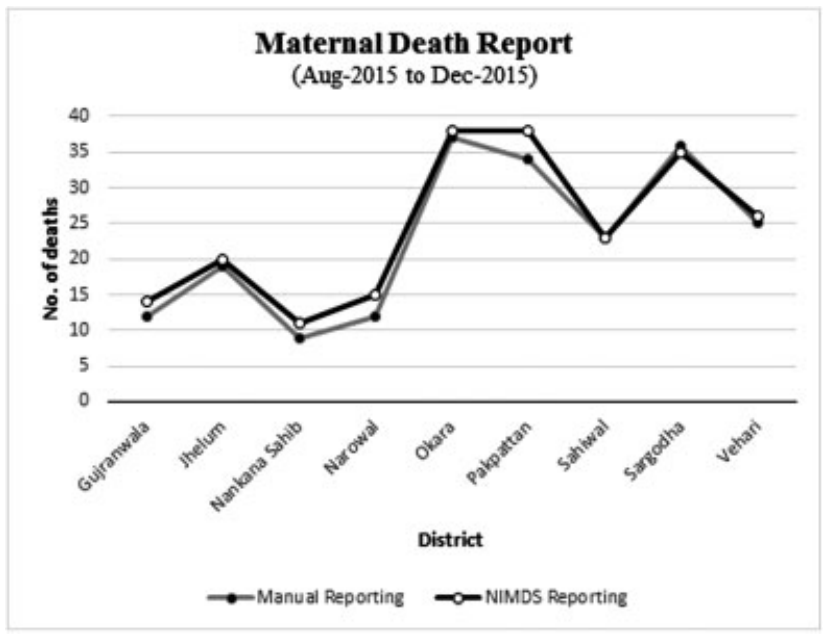

Fig. 8 District-wise maternal death comparison between manual reporting and Neonatal Infant and Maternal Deaths E-surveillance System (NIMDS) reporting.

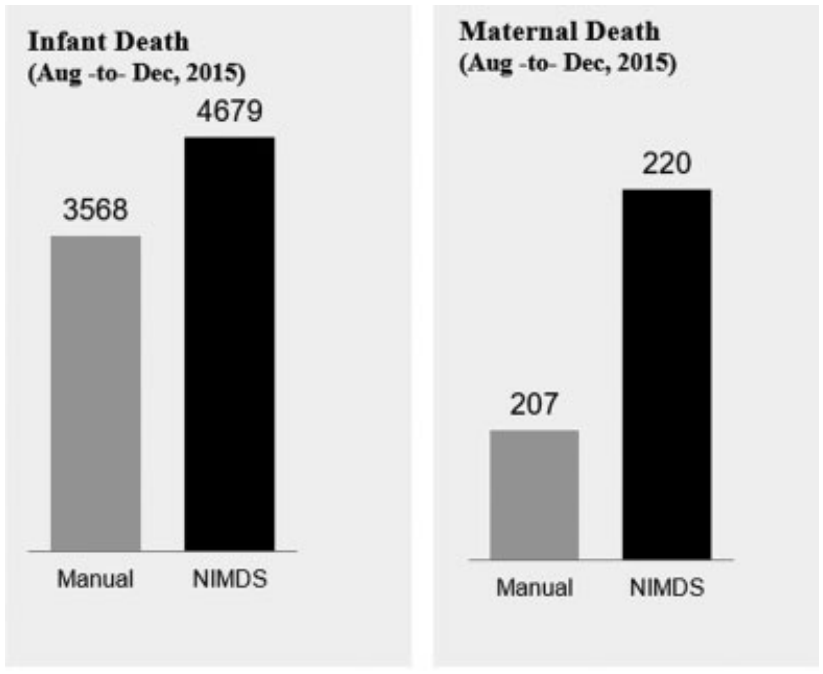

Fig. 9 Death reported cases in manual reporting and Neonatal Infant and Maternal Deaths E-surveillance System (NIMDS) reporting.

\section{Quality of Data}

The quality of data really matters for a quick response and correct decision making. NIMDS has specific protocols for a response with essential information to be filled up and verified by the field monitors. Consequently, the health department is able to provide quick response on the basis of real-time monitoring of deaths in each district.

\section{Centralized Repository}

In NIMDS, the reported data are at a central place where the information is kept and maintained in an organized way. This allows every department inside the organization to get access of the data in a unified way where permitting the higher management to access the real-time data for quick decision making.

\section{Accountability}

Old methods disguised the efforts of the LHSs as it was difficult to maintain true picture of the entire situation about infant and maternal health. Now tracking of individual cases and countermeasure strategies could be implemented efficiently.

\section{Discussion}

Pakistan may be divided into three regions with respect to health facilities. First, the country has developed cities with all modern health facilities. The second type of regions includes developing towns and modern villages with midsized health facilities in form of rural health centers (RHCs). The third type includes rural areas including the desert range of Cholistan, Thar, and Northern areas. In such rural areas, there are not sufficient health facilities. However, the network of LHSs, LHWs, and CMWs is accessible to most regions.

Currently, the health department provides health services through District Head Quarters, Tehsil Head Quarters, RHCs, and Basic Health Units hospitals. These services play a pivotal role in provision of reproductive maternal, child healthcare, and family planning services. The Punjab health department confirmed that on average a population of 1,400 to 1,500 residents in the rural areas and 1,500 to 1,600 in the urban areas are facilitated by one LHW responsible for primary healthcare and family planning services. There are around 46,000 LHWs in the Punjab province providing health services at the community level. The LHS is responsible for administrative and supportive supervision of LHWs in each health facility. The ratio of the LHSs to LHWs is around 1:25 and this ratio may be changed with respect to dynamic requirements based on the terrain and demography.

As per the result of our sessions, LHSs were found less comfortable with smartphone sage. Most of the LHSs were hesitant and did not know how to use a smartphone and play games on it because they were not familiar with. Another major concern about developing a smartphone app for LHSs was that in most of the rural areas there is no Internet coverage, which made it difficult for the LHSs to report from their respective health facilities. Developing countries like Pakistan and South Asian countries have heterogeneous 
pockets in terms of health facilities, living style, demography, and facilities. The metropolis areas of Lahore and Islamabad are varied from rural areas of desert like Cholistan and Thar. For example, there is variation in food and clean drinking water, health facilities, electricity, vaccination reachability, basic education standard for expecting mothers, and so on. These are some of the reasons due to which such areas form heterogeneous demographic pockets with respect to mortality causes.

\section{Conclusion}

There was a dire need of real-time reporting system for verbal autopsy in low-income countries like Pakistan. In these countries there are geographical pockets with high heterogeneity in all aspects for causes of neonatal, infant, child, and maternal mortality. Also, the manual registration process is flawed with many biases and a lot of layers of transformation that puts a question mark on the accuracy of the data maintained and used for research to achieve MDG and Sustainable Development Goal (SDG).

SDG cannot be achieved without first actually determining the causes of mortality to devise an effective countermeasure strategy. The contemporary situation in Pakistan for neonatal and maternal death in the context of MDG and SDG is not satisfactory. Pakistan is among those few countries of the world where maternal deaths are still increasing. It is evident that countermeasures by the WHO, the UN, and the Government of Pakistan proved ineffective with respect to MDG in last two decades. The result may not be expected different till 2030 for achieving the SDG. A positive step toward SDG may be realized by first developing and deploying a real-time reporting system for neonatal and maternal mortality.

This research helped achieving one milestone of gathering real-time mortality data from grassroot level using the UCD methodology. The identification of real causes of neonatal, infant, and maternal mortality will now become possible on actual primary data without any estimation theory used. Based on the quality data available, appropriate countermeasure policies will thus be adopted to achieve the SDG. The proposed system is the official death registration system for neonatal, infant, and maternal implemented in all 36 districts of Punjab, Pakistan. The success of NIMDS in the Punjab province of Pakistan will persuade governments in other provinces of Pakistan to adopt this system.

\section{Future Work}

The research on improving NIMDS is in progress. In the next phase, the NIMDS SMS system will be converted to voicebased input and response based on end user expectations in context of user-centered interaction and design. The voice input can be converted to the required text message with an appropriate format. The feasibility study is under progress on voice-based user-centered upgrade to NIMDS. The authors of this article are also interested to integrate global positioning system-based tracking in NIMDS so that real-time data on mapping of demographic pockets of mortality may also become available. These data will be useful for any shift in trends of mortality causes in various demographic pockets. This functionality will also be useful where GSM coverage is not available in rural areas in the world.

\section{Multiple Choice Questions}

1. What is the age range of a baby, to consider it under the category of neonatal mortality?

a. $<28$ days.

b. $<40$ days.

c. $<42$ days.

d. $<1$ year.

Correct Answer: The correct answer is option a. Age range must be below 28 days to consider the death of a baby under category of neonatal mortality.

2. All of the following are true about United Nations Millennium Development Goals except.

a. To promote gender equality and empower women.

b. To reduce child mortality.

c. To achieve universal higher education.

d. To improve maternal health.

Correct Answer: The correct answer is option c. It must be to achieve universal primary education instead of higher education.

Note

The data used to support the findings of this study are available from the corresponding author upon request and the data will be provided after departmental permission.

\section{Protection of Human and Animal Subjects}

This article does not contain any studies with human participants or animals performed by any of the authors.

\section{Conflict of Interest}

None declared.

\section{Acknowledgments}

We thank all who participated in our research specially IRMNCH \& Nutrition Program, Primary \& Secondary Healthcare Department, Punjab, Pakistan, and United Nations Population Fund (UNFPA), Pakistan.

\section{References}

1 Lawn JE, Cousens S, Zupan J; Lancet Neonatal Survival Steering Team. 4 million neonatal deaths: when? where? why?. Lancet 2005;365(9462):891-900

2 Government of Pakistan. P.C.: Pakistan millennium development goals report2013. A Report of the Government of Pakistan BlockP, Pakistan Secretariat, Islamabad, Pakistan;2013

3 Alkema L, Chou D, Hogan D, et al; United Nations Maternal Mortality Estimation Inter-Agency Group collaborators and technical advisory group. Global, regional, and national levels and trends in maternal mortality between 1990 and 2015, with scenario-based projections to 2030: a systematic analysis by the UN Maternal 
Mortality Estimation Inter-Agency Group. Lancet 2016;387 (10017):462-474

4 Victora CG, Requejo JH, Barros AJ, et al. Countdown to 2015: a decade of tracking progress for maternal, newborn, and child survival. Lancet 2016;387(10032):2049-2059

5 Demographic P. Health survey 2012-13. Islamabad, Pakistan, and Calverton, Maryland, USA: National Institute of Population Studies and ICF International;2013

6 Pasha $\mathrm{O}$, Saleem S, Ali S, et al. Maternal and newborn outcomes in Pakistan compared to other low and middle income countries in the global networks maternal newborn health registry: an active, community-based, pregnancy surveillance mechanism. Reprod Health 2015;12(02):1

7 Harrison MS, Ali S, Pasha O, et al. A prospective population-based study of maternal, fetal, and neonatal outcomes in the setting of prolonged labor, obstructed labor and failure to progress in lowand middle-income countries. Reprod Health 2015;12(02, Suppl 2):S9

8 Medhi I, Jain M, Tewari A, Bhavsar M, Matheke-Fischer M, Cutrell E. Combating rural child malnutrition through inexpensive mobile phones. In: Proceedings of the 7th Nordic Conference on Human-Computer Interaction: Making Sense Through Design. ACM;2012:635-644
9 Liu L, Oza S, Hogan D, et al. Global, regional, and national causes of child mortality in 2000-13, with projections to inform post-2015 priorities: an updated systematic analysis. Lancet 2015;385 (9966):430-440

10 Hogan MC, Foreman KJ, Naghavi M, et al. Maternal mortality for 181 countries, 1980-2008: a systematic analysis of progress towards Millennium Development Goal 5. Lancet 2010;375(9726):1609-1623

11 Ahmad N. Virtual character based interactive interfaces for deaf and functionally illiterate users. In: International Conference of Software Business. Springer;2013:223-224

12 Ahmad N. People centered HMI's for deaf and functionally illiterate users. PhD Thesis. Politecnico di Torino-Universität Potsdam, Germany; 2014

13 Ahmad N, Shoaib U, Prinetto P. Usability of online assistance from semiliterate users perspective. Int J Hum Comput Interact 2015; 31(01):55-64

14 Bureau of Statistics, G.o.P. Pakistan Social and Living Standards Measurement Survey (PSLM);2014-2015

15 Norman DA. The Design of Everyday Things: Revised and Expanded edition. New York: Basic Books; 2013

16 Abras C, Maloney-Krichmar D, Preece J. User-centered design. In: Bainbridge W, ed. Encyclopedia of Human-Computer Interaction. Vol. 37. Thousand Oaks: Sage Publications; 2004:445-456 